\title{
PREPARATION OF CHEMICAL AND PHYSICAL CONJUGATES OF SELF- ASSEMBLING NANOPARTICLES WITH CELL-PENETRATING PEPTIDE AND DOXORUBICIN
}

\author{
Zhadyra Sagykyzy Shagyrova ${ }^{1}$, Yerzhan Yersaiynuly Zhiyenbay ${ }^{2}$, Mikhail Sergeyevich
} Voikov $^{3}$, Alexander Vyacheslavovich Shustov ${ }^{4}$

\begin{abstract}
Nano-sized carriers can help to reduce toxicity and improve clinical efficacy of drugs. Virus-like particles (VLPs) are biocompatible and biodegradable self-assembling nanoparticles, which show great promise as carriers for substances for targeted delivery and controlled release. Either chemical conjugation of physical incorporation without formation of covalent bonds is possible to load substances of interest into VLPs.
\end{abstract}

Objectives: To produce VLPs from recombinant viral capsid protein $(\mathrm{HBcAg})$ and test feasibility of methods of formation of chemical and physical conjugates of VLPs with substances of pharmacological interest.

Methods: Virus-like particles composed from recombinant hepatitis B core antigen ( $\mathrm{HBcAg}$ ) were produced by recombinant expression in E.coli and purified by successive centrifugation through sucrose gradients. Peptide transportan 10 was synthesized and used for carbodiimide (EDC)-mediated conjugation to VLPs. Doxorubicin (DOX) was loaded into the nucleic acid-containing VLPs to form physical conjugate.

Results: VLPs with chemically attached moieties of cell-penetrating peptide transportan 10 were produced. The conjugate was examined in SDS-PAGE to confirm presence of conjugation products. Conjugation efficiency (molar ration peptide/protein in the conjugate) reaches 0.5:1 (i.e. 50\% of protein chains have one attached peptide moiety). The nucleic acid-containing VLPs can be loaded with the DOX forming stable non-covalent physical conjugate.

Conclusion: Recombinantly expressed VLPs allow easy attaching of small molecules making them a convenient platform to develop drug carriers.

UDC Classification: 57.089, DOI: http://dx.doi.org/10.12955/cbup.v3.630

Keywords: virus-like particles, drug carrier, targeting, conjugate, transportan, Doxorubicin

\section{Introduction}

Targeting systems for directed transport and controlled delivery of pharmaceutical substances turned out to be a breakthrough field in pharmacology. Nanoengineered vehicles, which accumulate in certain organs or tissues or penetrate through biological barriers, may be loaded with a therapeutic agent and release it in a desirable organ or defined place in a body. Nanocarrier formulations allow reducing of toxicity and improving of clinical efficacy of already known drugs. Cancer chemotherapeutics are often toxic to normal tissues. Anthracycline antibiotic doxorubicin (DOX) exhibits strong cytostatic effect and is most widely used antitumor drug. However, DOX damages normal tissues and has a narrow therapeutic window meaning that any increase of dosage above marginally cytostatic level is limited by the development of systemic toxicity, particularly cardiotoxicity and myelosuppression (Rahman, Joher, \& Neefe, 1986; Bally, Nayar, Masin, Cullis, \& Mayer, 1990). Attempts have been made to reduce toxicity while maintaining therapeutic efficacy by coupling the DOX to biocompatible polymers (Levi-Schaffer, Bernstein, Meshorer, \& Arnon, 1982; Kopecek, Rejmanova, Duncan, \& Lloyd, 1985; Zunino, Pratesi, \& Pezzoni, 1987) or encapsulating this drug into liposomes. Indeed, liposomal form of DOX (Doxil) has been used in clinics (Northfelt et al., 1998).

\footnotetext{
1 Zhadyra Sagykyzy Shagyrova, staff researcher, National Center for Biotechnology, Astana, Kazakhstan, argin90@mail.ru

${ }^{2}$ Yerzhan Yersaiynuly Zhienbay, research engineer, Nazarbayev University Research and Innovation System (NURIS), Nazarbayev University, Astana, Kazakhstan, yezhiyenbay@nu.edu.kz

${ }^{3}$ Mikhail Seregeyevich Voikov, staff researcher, Nazarbayev University Research and Innovation System (NURIS), Nazarbayev University, Astana, Kazakhstan, mikhail.voikov@nu.edu.kz

${ }^{4}$ Alexander Vyacheslavovich Shustov, PhD, head of laboratory, National Center for Biotechnology, Astana, Kazakhstan, shustov@biocenter.kz
} 
Virus-like particles (VLPs) are self-assembling nanoparticles composed from multiple copies of one or more recombinantly expressed viral structural proteins. Capsid proteins of viruses are naturally involved in compaction of the viral genomes and assembly of capsid-the central structural component of virion, which contains viral DNA or RNA. Capsid proteins of many viruses expressed in heterologous environment (i.e. in the absence of replicating viral genome) retain their natural ability to assemble into particles, morphologically similar to viral capsids (Plummer \& Manchester, 2011). Recombinantly expressed VLPs, while structurally similar to viruses, lack genetic information with replicative capacity and hence do not have the safety issues associated with whole viruses. VLPs are efficiently utilized in human vaccines (e.g., hepatitis B vaccines - Recombivax, Engerix B; and human papillomavirus types 16,18 vaccines-Gardasil, Cervarix). However, currently, VLPs are clearly underexplored as targeting vehicles to transport drugs. Attractive properties of the recombinantly expressed VLPs include the biocompatibility and biodegradability, and that they can be produced in large quantities. The solution-exposed surfaces of VLPs can be decorated with the cellular receptorinteracting molecules or the targeting peptides in order to render the VLPs with a capability of targeting specific cell types. Cell penetrating peptides (CPPs) present with the ability to penetrate into intracellular environment from intercellular space by overcoming a physical barrier of a plasma membrane. A number of known peptides exhibit tropism, i.e. they accumulate in a particular tissue (e.g. nervous tissue) or organ (e.g. newly formed blood vessels, neovasculature) upon being injected into the systemic circulation. Peptides with this property are called homing peptides (HPs). Attempts to utilize the peptide-decorated nanoparticles include TAT-liposomes (Qin et al., 2011), RVG peptidecoated nanoparticles (Hwang do et al., 2011), and penetrating-functionalized nanoparticles from biocompatible polymer (PEG-PLA) (Xia et al., 2012).

We elected to construct self-assembling nanoparticles in the form of VLPs decorated with one CPPtransportan, or carrying the chemically conjugated DOX. Hepatitis B capsid (core) protein ( $\mathrm{HBcAg}$ ) was chosen as a VLP carrier.

\section{Materials and Methods}

\section{Expression construct}

Genetically-engineered construct Zh2.pET-HBcAg is a plasmid based on the pET22 vector (Novagen) and contains an insert of the chemically synthesized gene encoding the full-length (183 aa) HBcAg. The HBcAg gene is inserted under the control of T7 RNA polymerase promoter allowing expression of the recombinant protein in E.coli strains which produce T7 RNA polymerase (e.g. BL21(DE3)). The expected expression product (recombinant $\mathrm{HBcAg}$ ) contains dimerization domain (aa 1-149) which is responsible for the formation of dimers and VLPs and protamine-like domain (aa 150-183) which binds nucleic acids (either DNA or RNA or both).

\section{Production of HBcAg VLPs in E.coli}

One liter cultures of E.coli strain BL21(DE3) transformed with the plasmid Zh2.pET-HBcAg were grown at $37^{\circ} \mathrm{C}$ with intensive shaking to optical density OD600 $=0.6-0.8$. Expression of the recombinant $\mathrm{HBcAg}$ was induced by addition of isopropyl- $\beta$-thiogalactopyranoside (IPTG) to $1 \mathrm{mM}$. Induced culture was incubated for four hours. Bacterial cells were pelleted at $6000 \mathrm{rpm}$ and then they were washed with water. Cells were resuspended in $20 \mathrm{ml}$ of lysis buffer (20\% sucrose; $20 \mathrm{mM}$ HEPES pH 7.5; 5 mM EDTA; 0,1\% Triton X-100) and after that lysing chemicals were added: $2 \mathrm{ml}$ of lysozyme $(10 \mathrm{mg} / \mathrm{ml}), 2 \mathrm{ul}$ of DNase $(10 \mathrm{mg} / \mathrm{ml}), 20 \mathrm{ul}$ of RNase $(10 \mathrm{mg} / \mathrm{ml}), 2$ ul of PMSF $(0.2 \mathrm{M})$. Mixture was incubated at room temperature for 1 hour. Complete lysis of cells was achieved by ultrasonication (10 pulses, each pulse 20 seconds long with 3-minute intervals between pulses for cooling down). Urea was added to a final concentration of $0.5 \mathrm{M}$ and the mixture was incubated for 30 
minutes on ice. Debris was removed by centrifugation $10000 \mathrm{rpm}$ for 20 minutes at $4^{\circ} \mathrm{C}$ and the supernatant was utilized for purification of the VLPs in sucrose gradients.

Gradient ultracentrifugation

$\mathrm{HBcAg}$ VLPs were purified by successive rounds of ultracentrifugation in sucrose gradients (10-60\%). Tubes for rotor Beckman SW28 were filled with sucrose solutions. Portions $(4.5 \mathrm{ml})$ of sucrose solutions prepared in $\mathrm{HN}$ buffer (20 mM HEPES pH 7.5; $150 \mathrm{mM} \mathrm{NaCl}$ ) with w/w concentrations of sucrose $60 \%, 50 \%, 40 \%, 30 \%, 20 \%$ and $10 \%$ were layered into tubes. The rest of the tube volume was filled with the clarified supernatant containing the HBcAg VLPs. Probes were centrifuged at 28000 $\mathrm{rpm}$ for 3 hours and 45 minutes at $20^{\circ} \mathrm{C}$. Gradients were fractionated into 12-13 fractions, each fraction $2.6 \mathrm{ml}$. SDS-PAGE was used to monitor for the presence of the HBcAg. Fractions containing the $\mathrm{HBcAg}$ were combined and dialyzed overnight against $2 \mathrm{~L}$ of the $\mathrm{HN}$ buffer to remove sucrose. The VLP solution was concentrated to volume $<10 \mathrm{ml}$ using the Vivaspin 6 ultrafiltration devices with $100 \mathrm{kDa}$ cut-off limit.

\section{Removal of endotoxin}

Detergent Triton X-114 was added (to 1\%) to solution of the VLPs. Mixture was chilled on ice for 5 minutes and mixed by vigorous vortexing. Samples were placed into water bath at $56^{\circ} \mathrm{C}$ for 5 minutes to allow phase separation. Probes were centrifuged for 7 seconds at $13200 \mathrm{rpm}$. Upper aqueous phase was aspirated from the droplet of detergent. Procedure of endotoxin extraction was repeated 2 more times.

\section{Transmission electron microscopy}

Drop of sample (10 ul) was placed on a sheet of parafilm and one copper formwar-carbon-coated grid was allowed to float on the drop for 5 minutes. The grid was taken from the drop and excess of liquid was removed by touching a piece of filter paper. Drop (10 ul) of phosphotungstic acid ( $\mathrm{pH}$ 6.5) was placed on parafilm and the grid with adsorbed sample was allowed to float on the drop of contrasting agent for 5 minutes. The grid was dried and examined under the Libra 120 (Zeiss) transmission electron microscope.

Synthesis of transportan 10

Cell-penetrating peptide transportan 10 (AGYLLGKINLKALAALAKKIL) was synthesized using standard Fmoc-based chemistry on CS136XT (CS Bio, CA, USA) peptide synthesizer. On the first step the amino groups of the rink amide resin were deprotected with a solution of $20 \% \mathrm{w} / \mathrm{v} 4$ methylpiperidine in DMF. Couplings were performed using 4-fold molar excess of each Fmoc Lamino acid, 4-fold molar excess of HBTU, and 8-fold molar excess of DIEA. Final deprotection and cleavage from the resin was carried out with trifluoroacetic acid (TFA), triisopropylsilane (TIPS) and water (TFA/TIPS/H2O 95/2.5/2.5). The resin beads were filtered, and the filtrate was precipitated in diethyl ether, dissolved in water, and lyophilized. The crude peptide was purified by RP-HPLC on a $100 \AA 5 \mu \mathrm{m} \mathrm{C18} \mathrm{XBridge} \mathrm{column} \mathrm{using} \mathrm{a} \mathrm{linear} \mathrm{water/acetonitrile} \mathrm{gradient} \mathrm{containing} 0.05 \%$ TFA by vol $(6 \mathrm{~mL} /$ minute, detection $215 \mathrm{~nm}, 0-40 \% \mathrm{~B}$ in 60 minutes $)$. Fractions were collected and lyophilized to produce $96 \mathrm{mg}$ of white fluffy peptide. The purity of peptide was $>95 \%$ as determined by analytical RP-HPLC. Purified transportan 10 was dissolved in water to conc. $10 \mathrm{mg} / \mathrm{ml}$.

Chemical linking of cell-penetrating peptide to VLPs

VLPs were decorated with the cell-penetrating peptide transportan 10. EDC 1-ethyl-3-(3dimethylaminopropyl) carbodiimide was used to form covalent bonds between the primary amine groups of peptide and carboxyl groups of the carrier protein $\mathrm{HBcAg}$. The conjugation reaction contained $200 \mathrm{ul}$ of $\mathrm{HBcAg}$ (solution in $\mathrm{HN}$ buffer, $2 \mathrm{mg} / \mathrm{ml}$ ), $200 \mathrm{ul}$ of transportan 10 (solution in 
water, $10 \mathrm{mg} / \mathrm{ml}), 250 \mathrm{mkl}$ of reaction buffer (0.1 MES pH 4.7) and $50 \mathrm{ul} \mathrm{EDC} \mathrm{solution} \mathrm{in} \mathrm{water} \mathrm{(10}$ $\mathrm{mg} / \mathrm{ml})$. The mixture was incubated at room temperature for 1 hour with occasional (every 10 minutes) brief ultrasonication (1 pulse, 20 seconds) to prevent flocculation and formation of precipitates. Upon completion of the conjugation reaction the mixture was diluted 10 times with the HN buffer and the product was concentrated with the Vivaspin 6 (100 kDa cut-off). Simultaneous removal of the uncoupled peptide was achieved.

\section{Preparation of physical conjugate of DOX to HBcAg VLPs}

DOX is an anthracycline that intercalates into dsDNA or partially double-starnded RNA. Upon expression in E.coli the full-length $\mathrm{HBcAg}$ having protamine-like domain is expected to produce VLPs which contain nucleic acids (mainly various RNAs of bacterial origin) in the internal cavity. Water solution of DOX $(2 \mathrm{mg} / \mathrm{ml})$ was mixed with VLPs $(2 \mathrm{mg} / \mathrm{ml})$ and the mixture was allowed to set for 15 minutes at room temperature. The physical conjugate having characteristic red color of DOX was purified from unbound DOX by several rounds of 10x dilution with HN buffer and subsequent concentration on the centrifugal concentrator with $100 \mathrm{kDa}$ cut-off. Two rounds of purification were sufficient to remove the unbound DOX.

\section{Results}

Figure 1 presents the results of the first round of purification of the HBcAg VLPs in sucrose gradient. The fractions \#10,11, and 12 were combined, dialyzed, concentrated and subjected to second round purification by centrifugation in gradient.

Figure 2 presents TEM microphotograph of the purified HBcAg VLPs. Spherical particles (VLPs) of the expected size $(32-34 \mathrm{~nm})$ were abundant in the sample of the purified $\mathrm{HBcAg}$.

Sample of chemical conjugate of transportan 10 to VLPs was subject to SDS-PAGE. EDC-mediated reaction leads to formation of a covalent bond between the peptide and the protein subunit of VLP (one $\mathrm{HBcAg}$ molecule). This bond (carboxamide, -CONH-) is stable in conditions used to prepare samples for the SDS-PAGE. Conjugation of one peptide molecule to the HBcAg leads to increase in Mw by $\sim 2 \mathrm{kDa}$. 15\% PAGE allows separation of unconjugated and conjugated HBcAg monomers which form distinct bands (Fig. 3). Lane loaded with the transportan/VLP conjugate contains two bands one of which (lower band) shows mobility characteristic to the control (unmodified monomeric) $\mathrm{HBcAg}$ and the second (upper band) has higher apparent molecular weight by $\sim 2 \mathrm{kDa}$ (Figure 3).

Figure 1: SDS-PAGE analysis of distribution of proteins from bacterial cell lysate in sucrose gradient. Lanes: M - protein molecular weight marker K973 (Amresco), respective molecular masses of marker's bands indicated; lanes 4-12 represent gradient fractions \#4-12. Arrow points at band of the $\mathrm{HBcAg}$ monomer (21 $\mathrm{kDa})$.

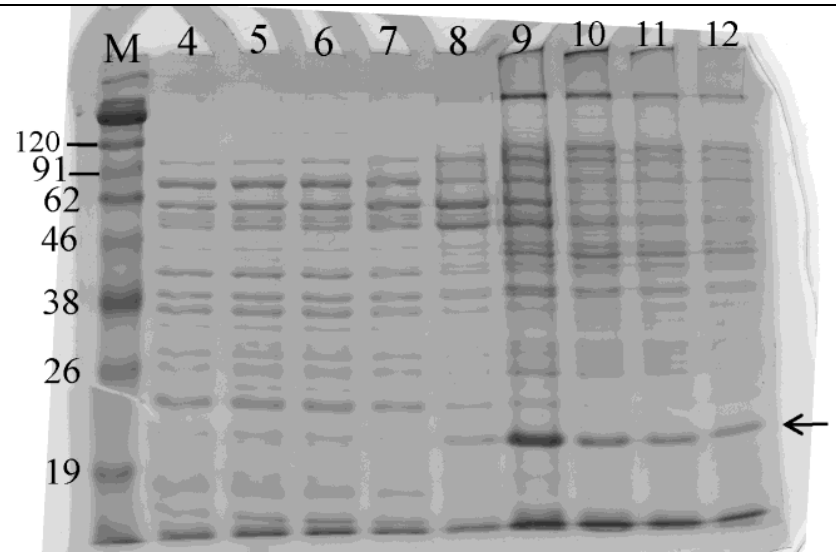

Source: Authors 


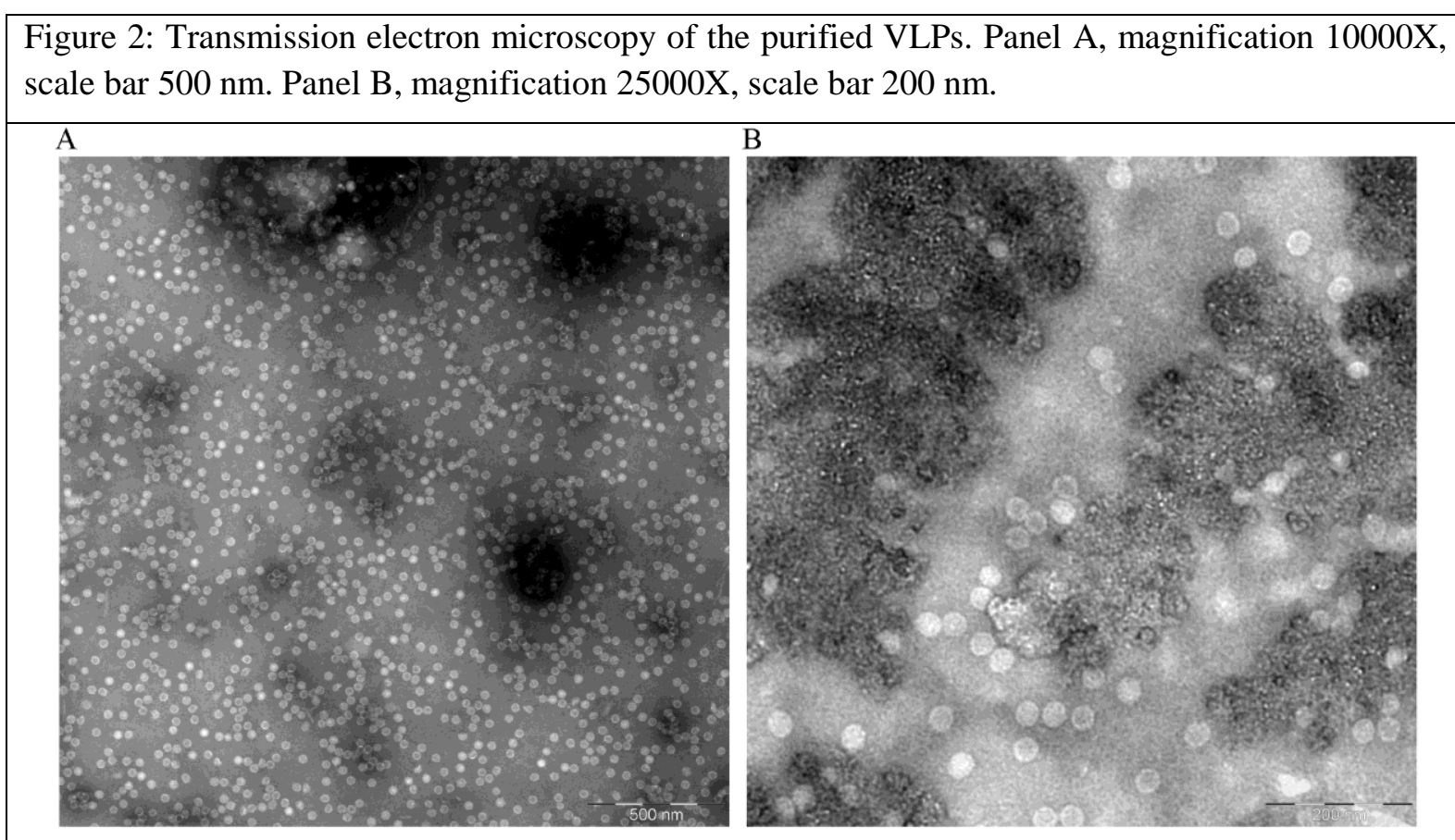

Source: Authors.

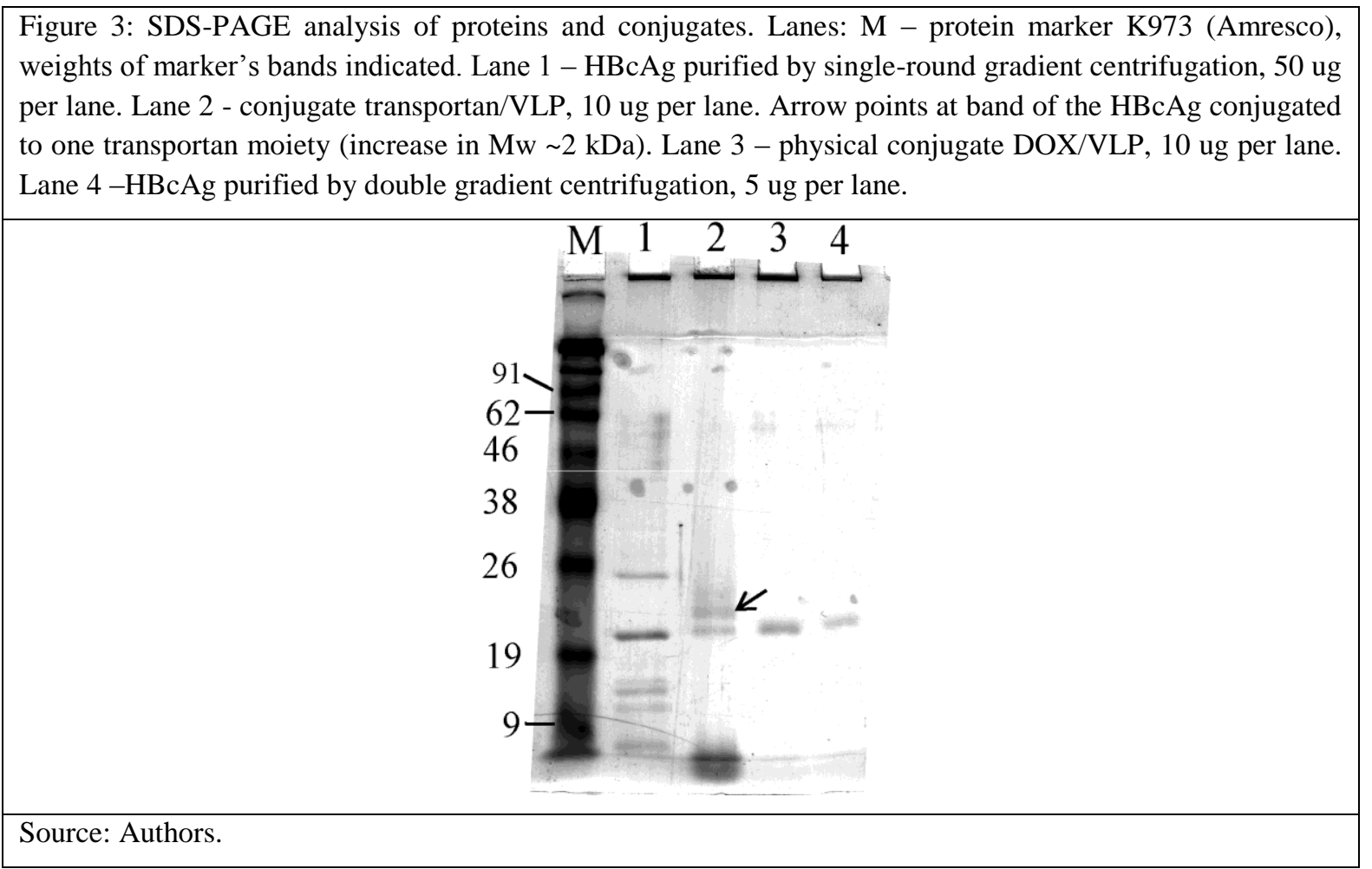

Relative intensity of these bands was quantified using TotalLab software. Based on intensity of these bands the resulting conjugate transportan/VLP has composition of the approx. 50\% of $\mathrm{HBcAg}$ molecules in unmodified form and the other $50 \%$ of protein chains having one attached peptide molecule. No cross-linking of two or more HBcAg moieties with formation of stable dimers/multimers was observed during conjugation reaction in described conditions.

Physical conjugate of DOX to VLPs was also loaded on the same gel for comparison. The concept of physical conjugate is different from chemical conjugate in that ligand is bound to carrier via the non- 
covalent bonds. No shift of the HBcAg bands was observed in SDS-PAGE in lanes loaded with the DOX/VLP (Figure 3). Physical conjugate of DOX to VLPs appeared to be stable during storage at $20^{\circ} \mathrm{C}$.

\section{Discussion}

In this report we describe production of self-assembling nanoparticles formed from recombinantly expressed protein and modification of these nanoparticles to serve as carriers for anticancer drug doxorubicin or surface-functionalization of the nanoparticles with cell-penetrating peptide. The virus like particles (VLPs) used in this study resemble viruses in that these particles demonstrate regular and uniform spatial structure, they are composed from exact number of subunits (T3 particle, 180 subunits; T4 particle, 240 subunits), each subunit represented by a single HBcAg chain. The presented VLPs also contain nucleic acids in their internal cavities. HBcAg is a protein having the VLP-forming capacity with the oldest history of utilization to create recombinant nanoparticles for vaccine and delivery applications (Whitacre, Lee, \& Milich, 2009).

We produced VLPs with attached peptide (CPP) to test feasibility of technology of chemical conjugation of the peptide (transportan 10) to the HBcAg. Published targeting systems utilizing superficial decoration of nanoparticles with CPPs include the use of HIV transactivator peptide (TAT), penetratin, TP, prion peptide, and SynB (Heitz, Morris, \& Divita, 2009; Fonseca, Pereira, \& Kelley, 2009; Gabathuler 2010; Gallo 2003; Kumar et al., 2007; Cai et al., 2011). Besides possibility of active targeting with use of ligand-receptor or membrane-receptor interactions, nanoparticles of certain sizes demonstrate preferential accumulation in tumors due to the so-called effect of enhanced permeability and retention (EPR) (Maeda, 2010). Chemotherapy agents utilizing the nano-sized carriers approved for clinical use include Doxil (liposomes with the diameter of $100 \mathrm{~nm}$ loaded with the antitumor drug doxorubicin, DOX) (Northfelt et al., 1998) and Abraxane (proteinaceous 130-nm nanoparticles loaded with paclitaxel) (Lohmann, Speers, \& Chia, 2013). We used different approach to load nanoparticles with the DOX. In our approach we use natural property of the DOX to bind to nucleic acids. DOX penetrates from solution into the internal cavities of VLPs and strongly binds to nucleic acids forming stable physical conjugate.

\section{Conclusion}

Recombinant DNA technology allows producing virus-like particles, which show potential for use as carriers for drugs and exert valuable properties, such as biodegradability, natural propensity to target particular tissues and cell types, and lack of systemic toxicity. Homing and cell-penetrating peptides, when present on surface of particles, may modulate preferential accumulation of the particles in target organs and ensure targeted transport of cargo particles into diseased cells. During further development of the project, the DOX-loaded and surface-functionalized virus-like particles will be used to study their ability to traffic the Doxorubicin into cancer cells in culture.

\section{References}

Bally, M. B., Nayar, R., Masin, D., Cullis, P. R., \& Mayer, L. D. (1990). Studies on the myelosuppressive activity of doxorubicin entrapped in liposomes. Cancer Chemotherapy and Pharmacology, 27, 13-19, doi: 10.1007/BF00689270

Cai, B., Lin, Y., Xue, X. H., Fang, L., Wang, N., \& Wu, Z. Y. (2011). TAT-mediated delivery of neuroglobin protects against focal cerebral ischemia in mice. Experimental Neurology, 227, 224-31. doi: 10.1016/j.expneurol.2010.11.009

Fonseca, S. B., Pereira, M. P., \& Kelley, S. O. (2009). Recent advances in the use of cell-penetrating peptides for medical and biological applications. Advanced Drug Delivery Reviews, 61, 953-64. doi: 10.1016/j.addr.2009.06.001

Gabathuler, R. (2010). Development of new peptide vectors for the transport of therapeutic across the blood-brain barrier. Therapeutic Delivery, 1, 571-586. doi:10.4155/tde.10.35 
Gallo, G. (2003). Making proteins into drugs: assisted delivery of proteins and peptides into living neurons. Methods in Cell Biology, 71, 325-338. doi: 10.1016/S0091-679X(03)01015-X

Heitz, F., Morris, M. C., \& Divita, G. (2009). Twenty years of cell-penetrating peptides: from molecular mechanisms to therapeutics. British Journal of Pharmacology, 157, 195-206. doi:10.1111/j.1476-5381.2008.00057.x

Hwang do, W., Son, S., Jang, J., Youn, H., Lee, S., Lee, D., Lee, Y. S., Jeong, J. M., Kim, W. J., \& Lee, D. S. (2011). A brain-targeted rabies virus glycoprotein-disulfide linked PEI nanocarrier for delivery of neurogenic microRNA. Biomaterials, 32, 4968-75. doi: 10.1016/j.biomaterials.2011.03.047

Kaplan, L. D., Du Mond, C., Mamelok, R. D., \& Henry, D. H. (1998). Pegylated-liposomal doxorubicin versus doxorubicin, bleomycin, and vincristine in the treatment of AIDS-related Kaposi's sarcoma: results of a randomized phase III clinical trial. Journal of Clinical Oncology, 16, 2445-2451.

Kopecek, J., Rejmanova, P., Duncan, R., \& Lloyd, J. B. (1985). Controlled release of drug model from N-[2-hydroxy-propyl] methacrylamide copolymers. Annals of the New York Academy of Sciences, 446, 93-104. doi: 10.1111/j.17496632.1985.tb18393.x

Kumar, P., Wu, H., McBride, J. L., Jung, K. E., Kim, M. H., Davidson, B. L., Lee, S. K., Shankar, P., \& Manjunath, N. (2007). Transvascular delivery of small interfering RNA to the central nrvous system. Nature, 448, 39-43. doi:10.1038/nature05901

Levi-Schaffer, F., Bernstein, A., Meshorer, A., \& Arnon, R. (1982). Reduced toxicity of daunorubicin by conjugation to dextran. Cancer Treatment Reports, 66, 107-114

Lohmann, A. E., Speers, C. H., \& Chia, S. K. (2013). Evaluation of the clinical benefits of nanoparticle albumin-bound paclitaxel in women with metastatic breast cancer in British Columbia. Current Oncology, 20, 97-103. doi: 10.3747/co.20.1256.

Maeda, H. (2010). Tumor-selective delivery of macromolecular drugs via the EPR effect: background and future prospects. Bioconjugate Chemistry, 21, 797-802. doi: 10.1021/bc100070g

Northfelt, D. W., Dezube, B. J., Thommes, J. A., Miller, B. J., Fischl, M. A., Friedman-Kien, A., Plummer, E. M., \& Manchester, M. (2011). Viral nanoparticles and virus-like particles: platforms for contemporary vaccine design. Wiley Interdisciplinary Reviews. Nanomedicine and Nanobiotechnology, 3, 174-196. doi: 10.1002/wnan.119

Qin, Y., Chen, H., Yuan, W., Kuai, R., Zhang, Q., Xie, F., Zhang, L., Zhang, Z., Liu, J., \& He, Q. (2011). Liposome formulated with TAT-modified cholesterol for enhancing the brain delivery. International Journal of Pharmacy, 419, 85-95. doi: 10.1016/j.ijpharm.2011.07.021

Rahman, A., Joher, A., \& Neefe, J. R. (1986) Immunotoxicity of multiple dosing regimens of free doxorubicin and doxorubicin entrapped in cardiolipin liposomes. British Journal of Cancer, 54, 401-408. doi:10.1038/bjc.1986.190

Whitacre, D. C., Lee, B. O., \& Milich, D. R. (2009). Use of hepadnavirus core proteins as vaccine platforms. Expert Review of Vaccines, 8, 1565-1573. doi: 10.1586/erv.09.121

Xia, H., Gao, X., Gu, G., Liu, Z., Hu, Q., Tu, Y., Song, Q., Yao, L., Pang, Z., Jiang, X., Chen, J. \& Chen, H. (2012). Penetratin-functionalized PEG-PLA nanoparticles for brain drug delivery. Int $J$ Pharm., 436, 840-50. doi: 10.1016/j.jpharm.2012.07.029

Zunino, F., Pratesi, G., \& Pezzoni, G. (1987). Increased therapeutic efficacy and reduced toxicity of doxorubicin linked to pyran copolymer via the side chain of the drug. Cancer Treatment Reports, 71, 367-373 